\title{
Editorial
}

\section{Signal Processing Technologies for Ambient Intelligence in Home-Care Applications}

\author{
Francesco G. B. De Natale, ${ }^{1}$ Aggelos K. Katsaggelos, ${ }^{2}$ Oscar Mayora, ${ }^{3}$ and Ying Wu $^{2}$ \\ ${ }^{1}$ Department of Information and Communication Technology, University of Trento, Via Sommarive 14, 38050 Trento, Italy \\ ${ }^{2}$ Department of Electrical and Computer Engineering, Northwestern University, 2145 Sheridan Road, Evanston, IL 60208-3118, USA \\ ${ }^{3}$ Multimedia, Interaction and Smart Environments Group, Create-Net International Research Center, Via Solteri 38, \\ 38100 Trento, Italy
}

Received 22 March 2007; Accepted 22 March 2007

Copyright () 2007 Francesco G. B. De Natale et al. This is an open access article distributed under the Creative Commons Attribution License, which permits unrestricted use, distribution, and reproduction in any medium, provided the original work is properly cited.

The possibility of allowing elderly and people with different kinds of disabilities to conduct a normal life at home and to achieve a more effective inclusion in the society is attracting more and more interest from both industrial and governmental bodies (hospitals, healthcare institutions, and social institutions).

Ambient intelligence technologies, supported by adequate networks of sensors and actuators, as well as by suitable processing and communication technologies, could be one of the enabling factors to achieve such an ambitious objective.

Recent researches demonstrated the possibility of providing constant monitoring of environmental and biomedical parameters, and the possibility to autonomously originate alarms, provide primary healthcare services, activate emergency calls, and rescue operations through distributed assistance infrastructures. Furthermore, proactive systems help the user to perform daily activities, stimulating a more active and healthy lifestyle, and supporting functional rehabilitation and preservation processes.

Although some products are already appearing on the market, several technological challenges connected with these applications are still open, ranging from the development of enabling technologies (hardware and software) to the standardization of interfaces, the development of intuitive and ergonomic human-machine interfaces, and the integration of complex systems in a highly multidisciplinary environment.

The objective of this special issue is to collect the most significant contributions and visions coming from both academic and applied research bodies working in this stimulating research field. This is a highly interdisciplinary field comprising many areas, such as signal processing, image processing, computer vision, sensor fusion, machine learning, pattern recognition, biomedical signal processing, multimedia, human-computer interfaces, and networking. The focus is primarily on ambient intelligence and home automation technologies, considered as basic tools to build smart environments providing advanced home-care services.

The possibility of continuously monitoring the elderly and automatically detecting emergency situations clearly represents one of the priorities in home-care. The paper "Event detection using "variable module graphs" for home care applications," by Amit Sethi et al. proposes a new paradigm to better exploit ubiquitous audio-visual capture devices used in home-care applications, with a special focus on surveillance and complex event detection. Their approach relies on variable/module (V/M) graphs, a recent extension of factor graphs. V/M graphs are used to bridge the semantic gap between the huge amount of data produced by the capture devices and the useful high-level concepts to be elaborated by the vision system. From the application viewpoint, the primary objective is surveillance of location for subject tracking as well as detection of irregular or anomalous behaviors. This is done automatically with minimal human involvement, with the system being trained to raise an alarm when an anomalous behavior is detected.

Similarly, the work by J.-S. Hu, and T.-M. Su, titled "Robust background subtraction with shadow and highlight removal for indoor surveillance," tackles the problem of monitoring a person in the home environment. In this case, the 
authors concentrate on the robust detection of foreground regions in complex indoor scenes, in the presence of illumination changes and dynamic backgrounds. These are typical conditions in ambient-assisted living infrastructures, where the environmental conditions cannot be strictly controlled, and the false alarm rate can become high. Their proposed approach achieves a robust background subtraction by suitably combining three models, namely the color-based probabilistic background model (CBM, based on a Gaussian mixture model), the gradient-based probabilistic background model (GBM, based on the short-term and long-term CBMs), and the cone-shape illumination model (CSIM, used to identify shadows and highlights).

N. P. Cuntoor and R. Chellappa further emphasize the behavioral analysis problem in their paper "Mixed-state models for nonstationary, multiobject activities." Here, the objective is to model and segment human activities in order to achieve a better knowledge on the actions performed by a subject, and how such actions are performed. The methodology developed by the authors to pursue this goal relies on a mixed state-space approach. The discrete-valued component of the mixed state represents higher-level behavior, while the continuous-state models the dynamics within behavioral segments. A set of behaviors is defined, based on generic properties of motion trajectories, and is used to characterize segments of activities. A Viterbi-based algorithm is used to detect boundaries between segments. The usefulness of the proposed approach for temporal segmentation and anomaly detection is illustrated in different contexts, including the UCF database of human actions.

If technologies enabling a timely response to harmful events are important, the possibility of preventing such events through an early analysis of dangerous behaviors would be even more attractive. The paper "The PARAChute project: remote monitoring of posture and gait for fall prevention," by David J. Hewson et al. describes the results achieved within a joint research project named PARAChute (Personnes Âgées et Risque de Chute), whose primary aim was to develop a methodology that enables the detection of an increased risk of falling in community-dwelling elderly. The main goal is to provide a remote noninvasive assessment for static and dynamic balance assessments and gait analysis. This is achieved by using a combination of two tools: balance assessment and gait analysis. The first is based on biomechanical tests (a force plate, providing a measure of the static and dynamic equilibria), while the second makes use of a vision system. The two subsystems perform local processing and can be remotely interconnected to medical and support networks.

Remote monitoring is also the main goal of the paper "Real-time transmission and storage of video, audio, and health data in energency and home care sitiuations," by Ivano Barbieri et al. In this case, the focus is put on the efficient transmission of large-bandwidth streams of audio-visual data for telemedicine applications (continuous monitoring and emergency handling). The proposed mobile communication system is based on the ITU-T H.323 multimedia terminal recommendation, suitable for real-time data/video/audio and telemedical applications. The audio and video codecs H.264 and G723.1, respectively, were implemented and optimized in order to obtain high performance on the system target processors. Furthermore, offline media streaming, and storage and retrieval functionalities were supported by integrating a relational database in the hospital central system. A key aspect of the developed prototype is the use of low-cost consumer electronics in order to ease the market penetration of potential products.

Finally, the problem of assuring the privacy to endusers is of fundamental importance in hom-ecare applications, which have to deal with extremely sensitive data such as personal video and voice, biomedical signals. This problem is considered in the paper by Datong Chen et al. "Tools for protecting the privacy of specific individuals in video." The authors address two problems: first the automatic identification of people with limited labelled data, and second the problem of obscuring a human body in the video with preserved structure and motion information. The automatic identification is achieved by a discriminative learning algorithm, using a robust face detection and tracking algorithm. The body obscuration is implemented through a novel method, which removes the appearance information of the people while preserving rich structure and motion information. A prototype system was tested in a nursing home environment, demonstrating the possibility of minimizing the risk of exposing the identities of protected people while ensuring the usability of captured data for activity/behavior analysis.

We believe this issue will serve the readers well for many years to come on this important application area.

\section{Francesco G. B. De Natale Aggelos K. Katsaggelos Oscar Mayora Ying Wu}

Francesco G. B. De Natale received the Laurea degree in electronic engineering in 1990, and the Ph.D. degree in telecommunications in 1994, both from the University of Genoa, Italy. In 1995-1996, he was a Visiting Professor at the University of Trento, Italy, and from 1996 to 1999, he was Assistant Professor at the University of Cagliari, Italy. At present, he is Full Professor of telecommunications at the University of Trento, where he coordinates the didactic activities of the B.S. and M.S. courses in telecommunications engineering. He is Deputy Head of the Department of Information and Communication Technologies, where he leads the research activities of the Multimedia Communications Lab. His research interests are focused on image and signal processing, with particular attention to multimedia data compression, processing, and transmission. He was General Cochair of the Packet Video Workshop in 2000, and Technical Program Cochair of the IEEE International Conference on Image Processing (ICIP) in 2005 and of the Multimedia Services Access Networks (MSAN) in 2005. He is also an Associate Editor of the ACM/Springer Wireless Networks Journal from 2006. In 1998, he was the corecipient of the IEEE Chester-Sall Best Paper Award. He is a Senior Member of IEEE. 
Aggelos K. Katsaggelos received the Diploma degree in electrical and mechanical engineering from the Aristotelian University of Thessaloniki, Greece, in 1979, and the M.S. and Ph.D. degrees both in electrical engineering from the Georgia Institute of Technology, in 1981 and 1985, respectively. He is currently Professor of EECS at Northwestern University, Director of the Motorola Center for Seamless Communica-

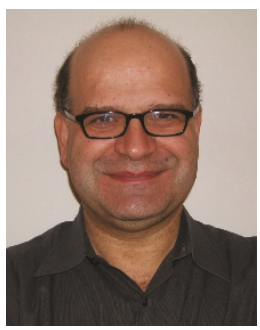
tions, and a Member of the Academic Affiliate Staff at Evanston Hospital. Dr. Katsaggelos is a member of the Publication Board of the IEEE Proceedings and a number of additional publications. He is the editor of Digital Image Restoration (SpringerVerlag, 1991), coauthor of Rate-Distortion Based Video Compression (Kluwer, 1997), coeditor of Recovery Techniques for Image and Video Compression and Transmission, (Kluwer, 1998), coauthor of Super-Resolution of Images and Video and Joint Source-Channel Video Transmission (both Morgan \& Claypool Publishers, 2007). $\mathrm{He}$ is the co-inventor of twelve international patents, a Fellow of the IEEE (1998), and the recipient of the IEEE Third Millennium Medal (2000), the IEEE Signal Processing Society Meritorious Service Award (2001), an IEEE Signal Processing Society Best Paper Award (2001), and an IEEE ICBE Best Paper Award (2006). He is a Distinguished Lecturer of the IEEE Signal Processing Society (2006-07).

Oscar Mayora obtained his B.S. degree in electronics and communications at Tecnológico de Monterrey, Mexico, in 1991. Later, he received an M.S. degree in computer science in the same institute and a Ph.D. degree in electronic engineering and informatics at DIBE, University of Genoa, Italy. In 2000, he joined the Advance Interactive Systems Laboratory at VTT Electronics in Oulu, Finland, as an ERCIM Visit-

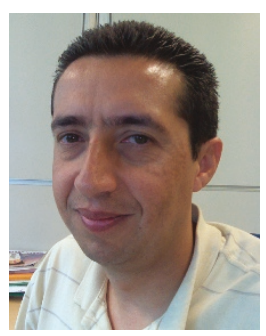
ing Research Fellow. In August 2001, he was appointed Associate Professor in the Computer Science Department at Tecnologico de Monterrey. In 2002, he became a Head of the Graduate Program In Computer Science at the same institution. Since September 2004, he is the Head of Multimedia, Interaction and Smart Environments Group in CREATE-NET International Research Center in Trento, Italy. His main research interests are in technologies for ambient intelligence and human-computer interaction.

Ying Wu received the B.S. degree from Huazhong University of Science and Technology, Wuhan, China, in 1994, the M.S. degree from Tsinghua University, Beijing, China, in 1997, and the Ph.D. in electrical and computer engineering from the University of Illinois at Urbana-Champaign (UIUC), Urbana, Ill, in 2001. From 1997 to 2001, he was a Research Assistant at the Beckman Institute for Advanced Science and Technology at UIUC. During summer 1999 and 2000, he was a Research Intern with Microsoft Research, Redmond, Washington. Since 2001, he has been an Assistant Professor at the Department of Electrical Engineering and Computer Science of Northwestern University, Evanston, Ill. His current research interests include computer vision, image and video analyses, pattern recognition, machine learning, multimedia data mining, and humancomputer interaction. He is an Associate Editor of SPIE Journal of Electronic Imaging and an Associate Editor of IAPR Journal of Machine Vision and Applications. He received the Robert T. Chien Award at UIUC in 2001, and the NSF Career award in 2003. He is a Senior Member of the IEEE. 